\title{
Research on fault diagnosis of planetary gearbox based on Variable Multi-Scale Morphological Filtering and improved Symbol Dynamic Entropy
}

Tongtong Liu ( $\sim$ liuzhaishi@emails.bjut.edu.cn )

Beijing University of Technology

Lingli Cui

Beijing University of Technology

Jianyu Zhang

Beijing University of Technology

Chao Zhang

Inner Mongolia University of Science and Technology

Research Article

Keywords: Planetary gearbox, Fault diagnosis, VMSMF, AMDSDE, Fault pattern recognition

Posted Date: September 9th, 2021

DOl: https://doi.org/10.21203/rs.3.rs-805447/v1

License: (c) (i) This work is licensed under a Creative Commons Attribution 4.0 International License. Read Full License

Version of Record: A version of this preprint was published at The International Journal of Advanced Manufacturing Technology on January 8th, 2022. See the published version at https://doi.org/10.1007/s00170-021-08085-0. 


\title{
Research on fault diagnosis of planetary gearbox based on Variable
}

\section{Multi-Scale Morphological Filtering and improved Symbol Dynamic}

\section{Entropy}

\author{
Tongtong liu ${ }^{1,2}$, Lingli Cui ${ }^{1}$, Jianyu Zhang ${ }^{1}$, Chao Zhang ${ }^{2}$
}

1 Key Laboratory of Advanced Manufacturing Technology, Beijing University of Technology, Chao Yang District, Beijing 100124, China

2 Inner Mongolia Key Laboratory of Intelligent Diagnosis and Control of Mechatronic Systems, Inner Mongolia University of Science and Technology, Baotou 014010, China

Corresponding author: liuzhaishi@emails.bjut.edu.cn

Abstract: Under complex working conditions with noise interference, the fault feature of planetary gearbox is difficult to be extracted and the fault mode is difficult to be identified. To tackle this problem, the technologies of Variable Multi-scale Morphological Filtering (VMSMF) and Average Multi-scale Double Symbolic Dynamic Entropy (AMDSDE) are proposed in this paper. VMSMF selects Chebyshev Window as the structural element and automatically selects the optimal scale parameters according to the signal characteristics of the planetary gearbox, which improves the filtering accuracy and calculation efficiency. AMDSDE fully considers the correlation between various state modes. Once combined with relevant knowledge of Mathematical statistics, the algorithm can effectively reduce misjudgment. Firstly, the Turn Domain Resampling (TDR) is used to transform the time domain signal of variable speed into the angle domain signal that is not affected by speed change. Secondly, the proposed VMSMF is used to de-noise the vibration signal, and the fault signal with a high signal-to-noise ratio is obtained. Finally, AMDSDE is used to extract the entropy value of the fault signal and judge the fault type. The proposed technology is verified by four kinds of signals collected from the sun gear of the planetary gearbox under non-stationary working conditions.

Keywords: Planetary gearbox, Fault diagnosis, VMSMF, AMDSDE, Fault pattern recognition

\section{Introduction}

When the transmission ratio is the same, the planetary gearbox has a smaller volume, less weight, and larger bearing capacity compared with the traditional fixed shaft gearbox. As planetary gearbox has a large transmission ratio, so it is widely used in aerospace, wind power generation, mining equipment, vehicles, and other transmission systems ${ }^{[1-3]}$. It is usually used in low-speed and heavy-duty unsteady environments. When the meshing force of each wheelset changes, the vibration and impact would change accordingly. The vibration signal of the planetary gearbox is the coupling of many kinds of excitation. The meshing signal includes the meshing between the sun gear and planetary gear and that between planetary gear and inner gear ring. Multiple meshing caused characteristic frequencies are difficult to be extracted. The periodic changes in the relative sensor position of planetary gear lead to the continuous changes of transmission path and distance of meshing signal, so the measured signal is the modulation one. The complex fault signal of the planetary gear system, especially the 
early weak fault, can be easily submerged by strong noise. Therefore, it is necessary to de-noise the early vibration signals so that the fault features can be highlighted.

Morphological filtering (MF) is a kind of nonlinear filtering method that retains the main morphological features. The method is simple, reliable, and effective, thus being widely used in vibration signal analysis of rotating equipment.

Scholars have successfully carried out a series of research on the filter scale and structural element selection. The concept of mathematical morphology was proposed by Serra and Matheron ${ }^{[4]}$ in the 1960s. In 2003, Nikolaou et al. ${ }^{[5]}$ first proposed the impact feature extraction algorithm of fault bearing based on the morphological filter operator. The algorithm analyzes the effect of basic morphological operators on the impulse signal, and studies the effect of physical attributes of structural elements on feature information extraction. In 2009, Wang et al. ${ }^{[6]}$ applied the pulse attenuation function to the bearing fault signal and obtained satisfactory results. However, the structural elements of this method are not targeted, and the filtering effect is limited. In 2013, Shen et al. ${ }^{[7]}$ defined structural elements through the local extreme value and the area of the time axis, and demonstrated the feasibility of this method by processing actual bearing fault signals. Jiang et al. ${ }^{[8]}$ improved the multi-scale morphological filtering algorithm and applied it to the fault signal analysis of hydraulic pumps. Li et al. ${ }^{\text {[9] }}$ evaluated the effect of 8 morphological operators in gear fault diagnosis based on the frequency coefficient. Chen et al. ${ }^{[10]}$ proposed a bearing fault classification method based on the multi-scale morphology of double-point structural elements. The abovementioned multi-scale algorithms need repeated calculation of each scale, reducing the computational efficiency. In order to solve the above problems, Cui et al. ${ }^{[11]}$ proposed to use the Chebyshev window function as a structural element in 2021 and carried out noise reduction analysis on fault signals of planetary gearbox, achieving good results.

The types of single tooth failures include broken teeth, missing teeth, cracks, pitting, etc. These faults cannot be effectively identified because the fault characteristic frequencies are similar. Entropy value change can be used to determine the fault types as it reflects the mutation behavior of dynamic characteristics of planetary gearbox. Besides, the corresponding entropy also varies with different dynamic characteristics of single tooth faults. Entropy theory was first proposed by C.E. Shannon ${ }^{[12]}$, and Shannon entropy laid a theoretical foundation for the subsequent study of entropy theory. Subsequently, Measure Entropy (K-S entropy) ${ }^{[13]}$, Approximate Entropy (AE) ${ }^{[14]}$, Sample Entropy (SE) ${ }^{[15]}$, and Permutation Entropy (PE) ${ }^{[16]}$ have been proposed, but they have some limitations in applications.

Symbolic time series analysis is a fast, simple and effective signal processing method based on symbolic dynamics, chaotic time series analysis and information theory ${ }^{[17]}$. It is used to transform the original time series into symbolic series with limited values, which is a coarse-granulation process ${ }^{[18,19]}$. Kurths et al. ${ }^{[20]}$ first proposed the concept of Symbolic Dynamic Entropy (SDE). Zhang et al. ${ }^{[21]}$ used the combination of SDE and SVM to accurately extract characteristics such as operation changes of vibration signals. The existing SDE method only considers the influence of state pattern vectors on fault characteristics, and the correlation between the state pattern vectors is not considered. To solve this problem, the improved SDE method, known as Double 
Symbolic Dynamic Entropy, is proposed in this paper. In the proposed method, the influence of the change of double sign before and after a specific state pattern vector on the entropy is proposed.

K-S entropy, SE, PE, and SDE can only be used to measure the complexity of time series from a single scale, but they cannot measure from other scales. Costa et al. ${ }^{\text {[2] }}$ introduced the scale factor and proposed the concept of Multi-scale Entropy (MSE). Ding et al. ${ }^{[23]}$ made SDE multi-scale. Li et al. ${ }^{[24]}$ proposed Multi-scale Symbolic Dynamic Entropy (MSDE), and applied this method to extract fault features of planetary gearbox, and achieved good results. The MSDE is developed based on SDE and it considers the influence of multiple scales on the signal at the same time, so it has obvious advantages for planetary gearbox signal processing. However, due to the complex working conditions of the equipment, the time domain signal includes various noise shocks, which will lead to the jump of entropy and affect its accuracy. Therefore, Multiscale processing of DSDE is carried out in this paper, and its entropy value is averaged. The Average Multi-Scale Double Symbolic Dynamic Entropy (AMDSDE) algorithm is proposed to avoid the impact of individual noise on the whole. The proposed method is used to process the fault signals of the sun gear of planetary gearbox, and good results are obtained.

The current morphological filtering algorithms mostly choose linear or triangular structural elements, which are not suitable for multiple impact signals such as planetary gearbox. Meanwhile, the multi-scale morphological filtering is repeated at each scale during the calculation, thus lowering the calculation efficiency. In current research on sign entropy, the signal is divided into several state modes according to its amplitude, and only the influence of the current state mode is considered when calculating entropy. However, aliasing may occur in the recognition of state modes due to the complex signal structure of planetary gearbox and the correlation between state mode signals. In addition, the existing symbolic entropy spectrum will fluctuate randomly due to the influence of noise and other interference factors, thus lowering the criterion effect. To address these problems, a fault pattern recognition method of planetary gearbox based on VMSMF and AMDSDE is proposed.

The Chebyshev window is selected as the structural element of VMSMF. The scale parameters of the structural element can be adjusted adaptively with the characteristics of vibration signals, thus making it more targeted and improving filtering precision. Before the signal is filtered, the signal is divided into several parts according to the characteristics of the impact, based on which, the appropriate scale is selected, thus avoiding the deficiency of multi-scale repeated calculation and improving the operation efficiency. In addition, AMDSDE proposes the concept of transfer mode, so it can consider the influence of the current state mode and neighboring mode simultaneously, making up for traditional entropy flaws. This method also takes into account that the entropy value obtained may be affected by noise and other interferences, and the entropy value of each scale is averaged. In this way, the interference fluctuation is reduced and the entropy spectrum curve can be smoother, so that each fault mode can be identified more easily. The main framework of the paper is shown in Fig. 1. 


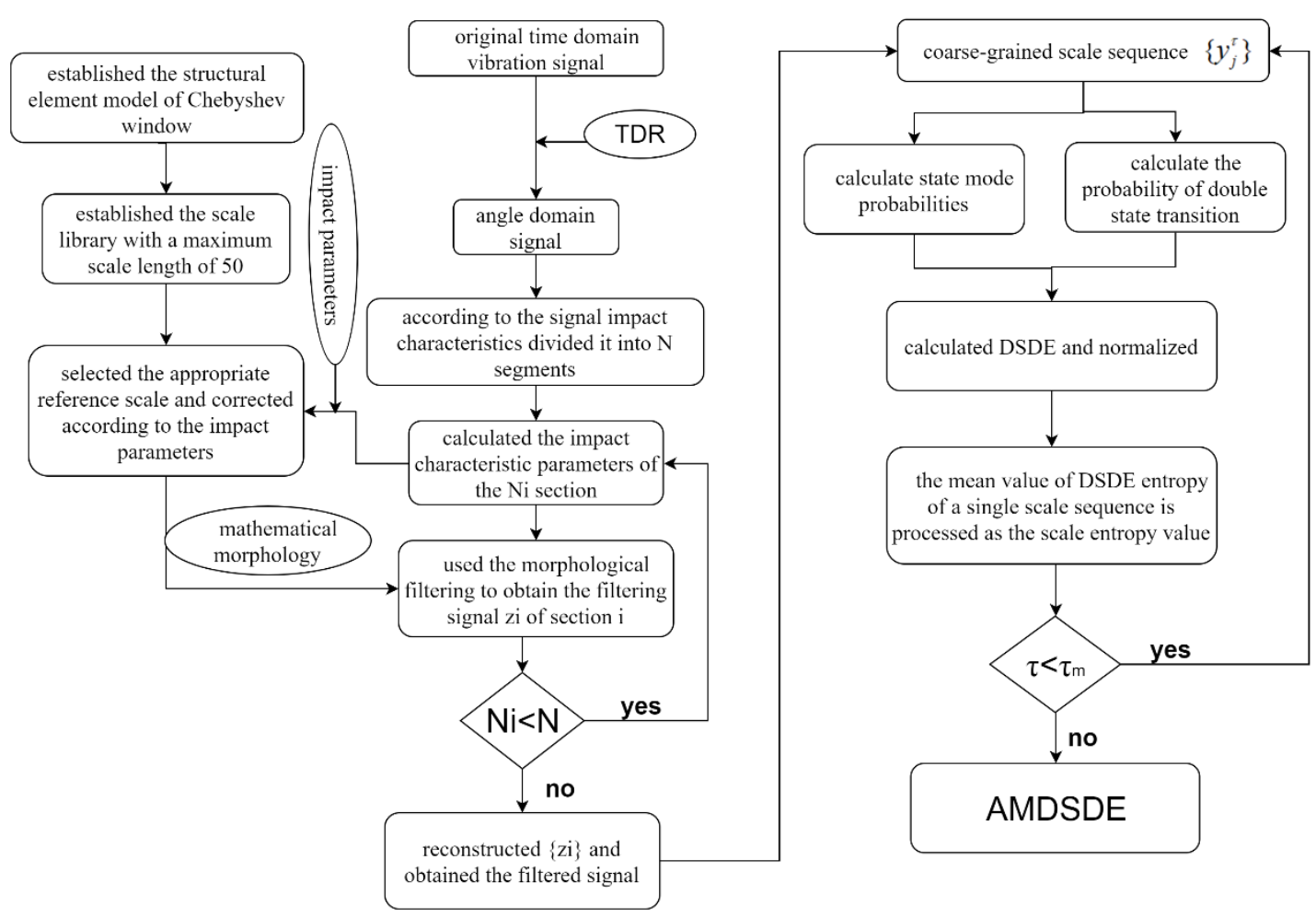

Fig. 1 Flow chart of vibration signal processing

The following part is arranged as follows. Section 2 introduces the basic theory of structural elements and morphological algorithm as well as the basic theory of variable multiscale morphological filtering algorithm and its application. In section 3, the calculation methods of the symbolic dynamical entropy and the average multi-scale double symbolic dynamic entropy are introduced. In Section 4, the VMSMF and AMDSDE are used to analyze four working conditions of planetary gearbox sun wheel. Finally, the main conclusions of this paper are summarized and the future research prospects are presented.

\section{Variable Multi-scale Morphological Filtering Algorithm Principle}

\subsection{Mathematical Morphological Algorithm}

\subsection{Structural Elements}

In mathematical morphology, structural elements are reference objects with special shapes, which are subjected to the influence of the morphological characteristics of original signals directly. The current structural element shapes are divided into flat and non-flat. The height of flat structural element is zero, which involves the maximum and minimum solutions. The scale of non-flat structural elements is determined based on the characteristics of the signal, and the morphological calculation is carried out by addition and subtraction methods. Then, the local extremum is solved. At present, the most commonly used structural elements include linear structural elements and triangular structural elements. For the former element, only the length is changed while the height is zero; for the latter element, both the length and height of triangular structure unit can change along with size, so its analysis effect is better. In addition, the length of triangular structural elements can only be odd, which is discontinuous in the length 
direction and cannot be accurately matched. Window function can be used as a structural element of morphological algorithm. There are two main indexes to evaluate window function as a structural element: (1) the width of main lobe should be as small as possible; (2) the height of side lobe should be as low as possible. This paper compares the main lobe and side lobe of rectangular window, triangular window, Hanning window, Hamming window, Blackman window, Chebyshev window and Duke window. The comparison results are shown in Fig. 2.

According to Figs. 2 (a) and (b), the Chebyshev window is the most ideal one. Therefore, the Chebyshev window is selected as the structural element of morphological algorithm. To further verify the robustness of the window function, the Hamming window, Hanning window, rectangular window, and Chebyshev window are used to filter 1000, 2000, 5000, 10000, 20000, and 40000 groups of data, respectively, and the correlation coefficients of the signal are calculated with and without filtering. The correlation data are shown in Table 1.
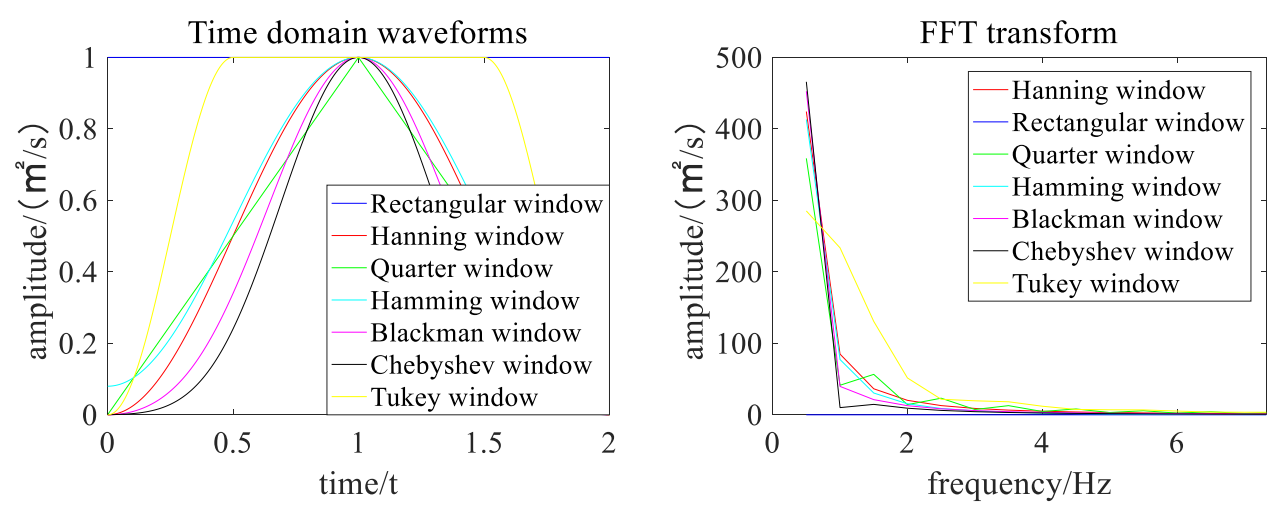

Fig. 2 Comparison of main / side lobe of each window function

Table 1 Correlation coefficients before and after morphological filtering of each window function

\begin{tabular}{ccccccc}
\hline $\begin{array}{c}\text { window } \\
\text { function }\end{array}$ & $\begin{array}{c}1000 \\
\text { groups }\end{array}$ & $\begin{array}{c}2000 \\
\text { groups }\end{array}$ & $\begin{array}{c}5000 \\
\text { groups }\end{array}$ & $\begin{array}{c}10000 \\
\text { groups }\end{array}$ & $\begin{array}{c}20000 \\
\text { groups }\end{array}$ & $\begin{array}{c}40000 \\
\text { groups }\end{array}$ \\
\hline Chebyshev & 0.7872 & 0.5732 & 0.7514 & 0.6658 & 0.6020 & 0.5658 \\
Hamming & 0.2235 & 0.2828 & 0.5943 & 0.5460 & 0.5310 & 0.5259 \\
Hanning & 0.1499 & 0.2807 & 0.5953 & 0.5458 & 0.5334 & 0.5297 \\
Rectangle & 0.0022 & 0.4002 & 0.4382 & 0.3117 & 0.2198 & 0.1551 \\
\hline
\end{tabular}

It can be seen from table 1 that using Chebyshev window as the structural element of morphological filtering, the stability of vibration signal after filtering is the best. Therefore, this paper finally selects Chebyshev window function as the structural element of morphological filtering.

$$
\omega(n)=(-1)^{n} \frac{\cos \left[(N-1) \operatorname{arcsch}\left[\beta \cos \left(\pi \frac{n}{N-1}\right)\right]\right]}{\cosh [(N-1) \operatorname{arcsch}(\beta)]} 0 \leq n \leq N-1
$$

where, $\alpha$ and $\beta$ are respectively:

$$
\beta=\cosh \left[\frac{1}{N-1} \operatorname{arcsch}\left(10^{\alpha}\right)\right]
$$




$$
\operatorname{ar} \operatorname{csch}(\beta)=\log \left(1+\operatorname{sqrt}(1-\beta)^{\wedge} 2 / \beta\right)
$$

In the equations above, $\mathrm{N}$ is the length of Chebyshev window, and $\alpha$ determines the attenuation degree of the side lobe of the window function. According to experience, $\alpha=4$ is selected.

The two key elements of structural elements are length and height and its parameters can directly affect the filtering effect. Because structural elements need to be used to strike a balance between noise removal and effective information suppression, signal characteristics need to be used to determine the structural element parameters of morphological filter. Based on existing research, the signal length between two adjacent shocks in the signal is extracted as the length, and the peak-to-peak value of the impact signal is calculated as the height. In this paper, a scale library of $n=50$ is established according to the sun gear signal characteristics of planetary gearbox. The scale library of Chebyshev structural elements is shown in Table 2.

Table 2 Chebyshev structural element scale library

\begin{tabular}{ccc}
\hline Scales & Length & Height \\
\hline 1 & 3 & $\mathrm{a}_{1}\left\{\omega_{1}(0), \omega_{1}(1), \omega_{1}(2),\right\}$ \\
2 & 4 & $\mathrm{a}_{2}\left\{\omega_{2}(0), \omega_{2}(1), \omega_{2}(2), \omega_{2}(3)\right\}$ \\
3 & 5 & $\mathrm{a}_{3}\left\{\omega_{3}(0), \omega_{3}(1), \omega_{3}(2), \omega_{3}(3), \omega_{3}(4)\right\}$ \\
$\ldots$ & $\ldots$ & \\
$\mathrm{n}$ & $\mathrm{n}+2$ & $\mathrm{a}_{\mathrm{n}}\left\{\omega_{\mathrm{n}}(0), \omega_{\mathrm{n}}(1), \omega_{\mathrm{n}}(2), \omega_{\mathrm{n}}(3), \ldots, \omega_{\mathrm{n}}(\mathrm{n}+1)\right\}$ \\
\hline
\end{tabular}

2.1.2 Mathematical Morphological Algorithm

The mathematical morphology has direct relation with geometry, which gives it a unique advantage in representing vibration signals. Therefore, it is used to represent and analyze signals from a geometric perspective ${ }^{[25-26]}$. Expansion and corrosion are two basic operations of mathematical morphology. Assume that the corresponding structural element of the certain original signal $f(n)(n=1,2 \ldots, N)$ is $g(m)(m=1,2,3, \ldots, M), N$ ? $M$. The expansion operator eliminates the positive pulse, and the negative pulse is sharper, while the corrosion operator eliminates the negative pulse, and the positive pulse is sharper ${ }^{[11]}$.

(1) Expansion

The expansion operation of $f(n)$ and $g(m)$, that is, $f \oplus g$ is defined as ${ }^{[27]}$ :

$$
(f \oplus g)(n)=\max _{m \in G}\{f(n-m)+g(m)\}
$$

(2) Corrosion

The corrosion operation of $f(n)$ and $g(m)$, that is, $f \Theta g$ is defined as ${ }^{[27]}$ :

$$
(f \Theta g)(n)=\min _{m \in G}\{f(n+m)-g(m)\}
$$

On the basis of the operations of expansion and corrosion, the open and close operations of mathematical morphology are expressed as:

$$
f \circ g(n)=f \Theta g \oplus g(n)
$$




$$
f g g(n)=f \oplus g \Theta g(n)
$$

where $\mathrm{o}$ and grepresent open operation and close operation, respectively. The open operator can remove the positive pulse and keep the negative pulse, while the opposite is true for the close operator. Maragos defines the morphological open-close and closeopen filters ${ }^{[28]}$.

$$
\begin{aligned}
& o c(f(n))=(f \circ g \mathrm{gg})(n) \\
& \operatorname{co}(f(n))=(f \circ g \circ g)(n)
\end{aligned}
$$

where, OC and CO represent open and close operations, respectively.

In order to suppress statistical bias, both morphological open-close mean combined filter and closed-open mean combined filter are used in this paper.

$$
y(n)=[\operatorname{co}(f(n))+o c(f(n))] / 2
$$

\subsection{Variable Multi-scale Morphological Filtering}

The single scale morphology has a single structural element in filtering fault signals, which is more obvious for single impact signal, but it is not applicable for the planetary gearbox fault signals that containing multiple groups of impact. Compared with single scale morphological algorithm, multi-scale morphology algorithm has stronger comprehensive analysis ability of signal. However, the computational efficiency reduces due to repeated calculation at various scales. To address this issue, the variable multi-scale morphological filtering method is proposed. The basic idea of this method is to select the appropriate structural elements based on the different impacts of planetary gearbox fault signals, which not only ensures the filtering accuracy, but also improves the calculation efficiency. The specific steps of this method are as follows:

(1) The Chebyshev window is selected as the structural element, and the scale library with scale length of 1-50 is established for future use.

(2) All the extreme points of the vibration signal to be processed are calculated, and the number of all the maximum points and minimum points are calculated, which are expressed as $\mathrm{Nm}$ and $\mathrm{Ni}$, respectively. The number of vibration signals between two adjacent extreme points is calculated according to the position of the extreme points.

(3) According to the number of extremum points obtained in the second step, the original vibration signal is cut off thoroughly. The specific distribution of extremum points is shown in Fig. 3:

(1) $\mathrm{Ni}-\mathrm{Nm}=1$, the data between the small value points of two adjacent poles are taken as the shock signal; the appropriate structural element scale parameters are selected according to the shock characteristics for calculation;

(2) Ni-Nm=-1, remove the data beyond the first and last two minimum values and then filter and analyze the vibration signal in turn.

(3) $\mathrm{Ni}=\mathrm{Nm} \&$ the minimum occurs first, the data after the tail minimum point are removed, and then the vibration signal is filtered and analyzed successively.

(4) $\mathrm{Ni}=\mathrm{Nm} \&$ the maximum occurs first, the data before the first minimum point are 
removed, and then the vibration signal is filtered and analyzed successively.

(a)

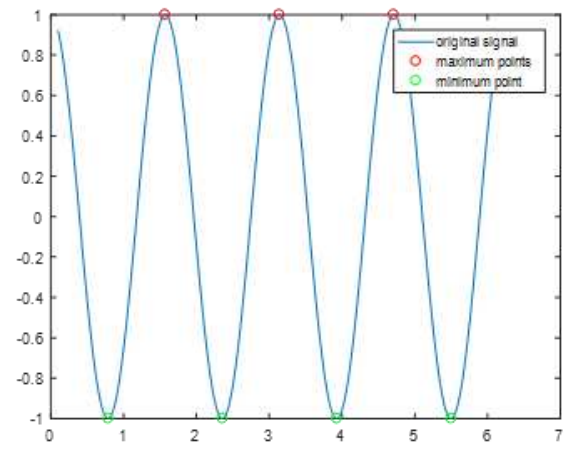

(c)

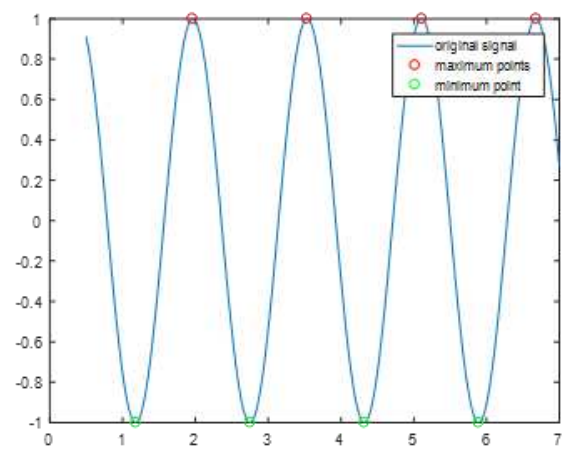

(b)

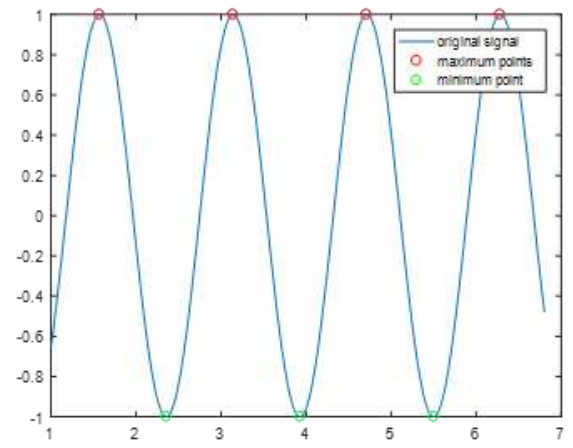

(d)

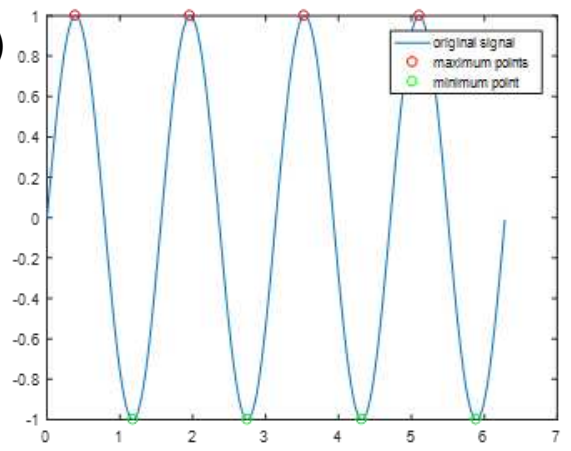

Fig.3. Distribution of extreme points of time-domain signals: (a) one more minimum point than maximum point (b) the minimum point is one less than the maximum point (c) the minimum point first appears when the minimum point and the maximum point are the same (d) the maximum point appearing first when the minimum point and the maximum point are the same

(4) For the $k$-th impact of the original signal, the number of discrete points between the two minimum points corresponding to the impact signal is selected as the length coefficient of Chebyshev window structure elements, as shown in Fig. 4.

(5) For the $k$-th impact of the original signal, the mean value of the two-pole small value is calculated as the minimum value, and the difference between the maximum and minimum value of the impact signal is taken as the height coefficient of the structural element.

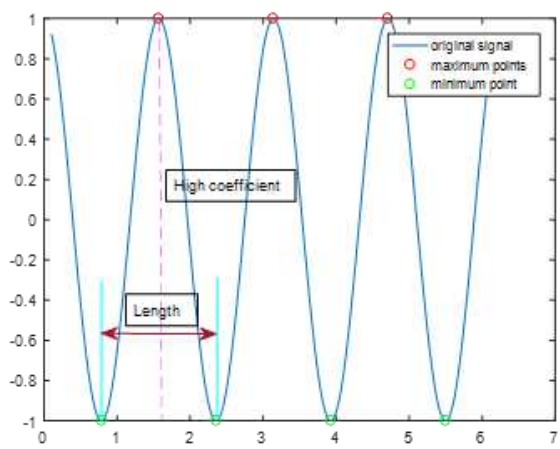

Fig.4 Schematic diagram of parameter selection of structural elements in VMSMF

(6) According to the characteristic parameters of the impact signal calculated in Step 
4 and Step 5, appropriate structural elements are selected to filter the vibration signal.

(7) Repeat steps 4 6 to analyze the vibration signals of each segment in turn. After the filtering is completed, the filtering signals of all impact segments are reconstructed to generate the filtering signals.

\subsection{Comparison of computational efficiency between VMSMF and MSMF}

In order to verify the advantages of the proposed method in terms of computing efficiency, the paper calculated 10,000, 20,000, 30,000, 40,000 and 50,000 simulation data using VMSMF and traditional MSMF algorithm. To prevent accidental errors when calculating each group of data, the data of each group are calculated 10 times respectively and the calculated average value is taken as its running time. The running time of the two methods is shown in Table 3.

Table 3 Processing elapsed time of data by using VMSMF and MSMF

\begin{tabular}{cccccc}
\hline data points & 10000 & 20000 & 30000 & 40000 & 50000 \\
\hline VMSMF & 1.7012 & 1.9718 & 2.2477 & 2.5684 & 2.888 \\
MSMF & 4.3347 & 8.7595 & 11.3497 & 14.0071 & 16.8224 \\
$\frac{M S M F}{V M S M F}$ & 2.55 & 4.44 & 5.05 & 5.45 & 5.83 \\
\hline
\end{tabular}

\section{Average Multi-scale Double Symbolic Dynamic Entropy}

\subsection{Traditional Symbolic Dynamics Entropy}

A time domain signal sequence $\{\mathrm{Z}\}=\left\{\mathrm{Z}_{1}, \mathrm{Z}_{2}, \mathrm{Z}_{3} \ldots, \mathrm{Z}_{\mathrm{N}}\right\}$ is supposed to be the sun wheel fault signal of planetary gearbox with a length of $\mathrm{N}$. The main parameters of the symbolic dynamics entropy in the calculation process are the sign number $\mathrm{S}$, the embedding dimension $\mathrm{M}$, and the time delay $\lambda$. The calculation procedure is mainly divided into three steps:

(1) Symbolize the time domain signal. The time domain signal is divided into s intervals according to the principle of average amplitude division. The corresponding amplitude interval is shown in Equation 11:

$$
\begin{aligned}
& \left\{\left[A_{\min }, A_{\text {min }}+\frac{1}{s}\left(A_{\text {max }}-A_{\text {min }}\right)\right),\left[A_{\text {min }}, A_{\text {min }}+\frac{2}{s}\left(A_{\text {max }}-A_{\text {min }}\right)\right),\right. \\
& \left.\mathrm{L},\left[A_{\text {min }}, A_{\text {min }}+\frac{s}{S}\left(A_{\text {max }}-A_{\text {min }}\right)\right]\right\}
\end{aligned}
$$

The symbols corresponding to each interval of amplitude are $\sigma_{i}(i=1,2, \mathrm{~L}, s)$ respectively. All elements in the time domain signal correspond to the amplitude interval one by one according to their amplitude, and assign values to the time domain signal according to the symbols corresponding to the corresponding interval. Finally, the corresponding symbol sequence can be obtained. The newly obtained symbol sequence is as follows:

$$
Y\left\{y_{n}(k), n=1,2, \mathrm{~L}, N, k=1,2, \mathrm{~L}, s\right\}
$$

where, $x_{n}(k)$ represents the symbol $s_{k}$ corresponding to the $\mathrm{n}$-th point of the original time domain signal. 
(2) Construct the state pattern matrix and calculate the state pattern probability. According to the embedding dimension $\mathrm{m}$ and time delay $\lambda$, the symbol sequence can be divided into $N-(m-1) \lambda$ sub vectors $Y_{i}^{m, \lambda}\{y(i), y(i+\lambda), \mathrm{L}, y(i+(m-1) \lambda)\}$, and the state pattern matrix is obtained as shown in Equation 13.

$$
Y_{i}^{m, \lambda}=\left[\begin{array}{cccc}
y_{1}(k) & y_{1+\lambda}(k) & \mathrm{L} & \left.y_{1+(m-1) \lambda}(k)\right) \\
y_{2}(k) & y_{2+\lambda}(k) & \mathrm{L} & y_{2+(m-1) \lambda}(k) \\
\mathbf{M} & \mathbf{M} & \mathbf{M} & \mathbf{M} \\
y_{N-(m+1) \lambda}(k) & y_{N-m \lambda}(k) & \mathrm{L} & y_{N}(k)
\end{array}\right] k \in[1,2, \mathrm{~L}, s]
$$

Potential symbol arrangement state patterns $s^{m}$ can be obtained from the symbol number and embedding dimension. According to the parameters selected by SDE, there are a total of $N-(m-1) \lambda$ permutation state modes in the above symbol sequence. The number of occurrences of each arrangement state mode is calculated by Equation 14 and the probability $q_{a}^{s, m, \lambda}$ is calculated, and it is recorded as $P\left(q_{a}^{s, m, \lambda}\right)$.

$$
P\left(q_{a}^{s, m, \lambda}\right)=\frac{\|\left\{i: i \leq N-(m-1) \lambda, \text { type }\left(Y_{i}^{m, \lambda}\right)=q_{a}^{s, m, \lambda}\right\} \|}{N-(m-1) \lambda}
$$

where, type $(\cdot)$ represented the mapping from symbol sequence to state pattern matrix, $\|\cdot\|$ is the norm of the set.

The state mode probability matrix $\left[P\left(q_{1}^{s, m, \lambda}\right), P\left(q_{2}^{s, m, \lambda}\right), \mathrm{L}, P\left(q_{s^{m}}^{s, m, \lambda}\right)\right]$ is constructed with the obtained state mode probability. From its characteristic, it can be known that $\operatorname{sum}\left(P\left(q_{a}^{s, m, \lambda}\right)\right)=1$, and the accuracy of the calculation process can be verified according to this characteristic.

(3) The SDE is calculated and normalized. According to the definition of Shannon entropy in information theory, SDE is defined as the cumulative value of the logarithm product of the probability of each state mode. Because the probability value of state mode is a decimal between 0 and 1, the logarithm value is negative, so the opposite number of SDE value is taken.

$$
\operatorname{SDE}(Z, s, m, \lambda)=-\sum_{a=1}^{s^{m}} P\left(q_{a}^{s, m, \lambda}\right) \log \left(P\left(q_{a}^{s, m, \lambda}\right)\right)
$$

It can be seen from Equation 14 that when the probabilities of all state modes are equal $\left(P\left(q_{a}^{s, m, \lambda}\right)=1 / s^{m}\right)$, the symbolic dynamics entropy reaches the maximum value, as shown in Equation 16.

$$
\left.\operatorname{SDE}(Z, s, m, \lambda)_{\max }=-\sum_{a=1}^{s^{m}} \frac{1}{s^{m}} \log \left(\frac{1}{s^{m}}\right)\right)=-\log \left(\frac{1}{s^{m}}\right)=\log \left(s^{m}\right)
$$

The SDE can be normalized according to Equations 15 and 16, as shown in Equation 17.

$$
\operatorname{SDE}(Z, s, m, \lambda)=\operatorname{SDE}(Z, s, m, \lambda) / \log \left(s^{m}\right)
$$

3.2 Double Symbolic Dynamic Entropy

Symbol sequence can be considered as a series of continuous state patterns. The 
traditional symbolic dynamics information entropy only considers the change of entropy under each state pattern. However, it ignores the correlation between each state pattern, which means it does not consider the influence of the changes of elements before and after a state pattern. After each sampling frequency corresponding to the time, the element symbol will move back slightly. Similarly, the state mode component will become the next state mode component, which is called the state transition. The influence of symbols of the elements before and after a state mode component is considered, namely, the occurrence of each symbol in the two adjacent elements of a certain state pattern is counted, and the state transition probabilities $P_{p r e}\left(\sigma_{b} \mid q_{a}^{s, m, \lambda}\right)$ and $P_{p o s t}\left(\sigma_{c} \mid q_{a}^{s, m, \lambda}\right) \quad$ are calculated, respectively. Then the state transfer probability matrix is obtained according to the pre-state and post-state transfer probability.

$$
\begin{aligned}
& \operatorname{SDE}(Z, s, m, \lambda)_{p r e}=-\sum_{a=1}^{s^{m}} \sum_{b=1}^{s} P\left(q_{a}^{s, m, \lambda}\right) P\left(\sigma_{b} \mid q_{a}^{s, m, \lambda}\right) \log \left(P\left(\sigma_{b} \mid q_{a}^{s, m, \lambda}\right)\right) \\
& \operatorname{SDE}(Z, s, m, \lambda)_{p o s t}=-\sum_{a=1}^{s^{m}} \sum_{c=1}^{s} P\left(q_{a}^{s, m, \lambda}\right) P\left(\sigma_{c} \mid q_{a}^{s, m, \lambda}\right) \log \left(P\left(\sigma_{c} \mid q_{a}^{s, m, \lambda}\right)\right)
\end{aligned}
$$

According to the pre and post state transfer probability matrix, the pre and post transfer symbol entropy are calculated, which are denoted as $S D E_{p r e}$ and $S D E_{p o s t}$, respectively.

$$
\begin{aligned}
& \operatorname{SDE}(Z, s, m, \lambda)_{p r e}=-\sum_{a=1}^{s^{m}} \sum_{b=1}^{s} P\left(q_{a}^{s, m, \lambda}\right) P\left(\sigma_{b} \mid q_{a}^{s, m, \lambda}\right) \log \left(P\left(\sigma_{b} \mid q_{a}^{s, m, \lambda}\right)\right) \\
& \operatorname{SDE}(Z, s, m, \lambda)_{p o s t}=-\sum_{a=1}^{s^{m}} \sum_{c=1}^{s} P\left(q_{a}^{s, m, \lambda}\right) P\left(\sigma_{c} \mid q_{a}^{s, m, \lambda}\right) \log \left(P\left(\sigma_{c} \mid q_{a}^{s, m, \lambda}\right)\right)
\end{aligned}
$$

Finally, the double-SDE is obtained as follows:

$$
\begin{aligned}
\text { double_SDE }(Z, s, m, \lambda)= & \operatorname{SDE}(Z, s, m, \lambda)_{\text {pre }}+\operatorname{SDE}(Z, s, m, \lambda) \\
& +\operatorname{SDE}(Z, s, m, \lambda)_{\text {post }}
\end{aligned}
$$

Similarly, double-SDE is normalized. If and only if all state mode probabilities and double state transfer probabilities are equal: $\left(P\left(q_{a}^{s, m, \lambda}\right)=1 / s^{m}, P_{p r e}\left(\sigma_{b} \mid q_{a}^{s, m, \lambda}\right)=1 / s, P_{p o s t}\left(\sigma_{c} \mid q_{a}^{s, m, \lambda}\right)=1 / s\right)$, the SDE reaches the maximum.

$$
\begin{aligned}
& \text { double_SDE }(Z, s, m, \lambda)_{\max } \\
& \left.\left.\left.=-\sum_{a=1}^{s^{m}} \frac{1}{s^{m}} \log \left(\frac{1}{s^{m}}\right)\right)-\sum_{b=1}^{s} \frac{1}{s} \log \left(\frac{1}{s}\right)\right)-\sum_{c=1}^{s} \frac{1}{s} \log \left(\frac{1}{s}\right)\right) \\
& =-\log \left(\frac{1}{s^{m}}\right)-\log \left(\frac{1}{s}\right)-\log \left(\frac{1}{s}\right)=\log \left(s^{m+2}\right)
\end{aligned}
$$


The double-SDE is normalized according to Equation 23, as shown in Equation 24.

$$
\text { double_SDE }(Z, s, m, \lambda)=\text { double_SDE }(Z, s, m, \lambda) / \log \left(s^{m+2}\right)
$$

3.3 Average Multi-scale Double Symbolic Dynamic Entropy

3.3.1 Traditional Multi-scale Symbolic Dynamical Entropy

Costa, M. ${ }^{[29]}$ initiated the concept of multi-scale analysis to measure the dynamic characteristics of time series on different scales. Because of its advantages, the Multiscale Symbolic Dynamic Entropy (MSDE) has been used in gearbox fault diagnosis and achieved good results. The calculation process of MSDE is mainly divided into the following two steps:

(1) The original time domain signal is segmented by coarse granulation. A time domain signal sequence $\left\{Z_{i}\right\}=\left\{z_{1}, z_{2}, z_{3}, \ldots, z_{N}\right\}$ is assumed to be the fault signal of planetary gearbox sun wheel of length $\mathrm{N}$, and the time domain signal is scaled by coarse-grained segmentation. Assuming that the scale factor is $\tau$, the time domain sequence $Y=\left\{y_{j}^{\tau}\right\}$ of each scale can be obtained, and its expression is as follows:

$$
y_{j}^{\tau}=\frac{1}{\tau} \sum_{i=j}^{j+\tau-1} x_{i} \quad 1 \leq j \leq N-\tau+1
$$

The time series of different scales are obtained by adjusting the scale factor $\tau$. Obviously, the time series obtained when $\tau=1$ is the same as the original time series.

(2) The SDE of time series $\left\{y_{j}^{\tau}\right\}$ at different scales is calculated, respectively.

$$
\operatorname{MSDE}(Z, s, m, \lambda, \tau)=\operatorname{SDE}\left(y_{j}^{\tau}, s, m, \lambda\right)
$$

\subsubsection{Average Multi-scale Double Symbolic Dynamic Entropy (AMDSDE)}

In order to verify the superiority of the multi-scale double symbolic dynamic entropy (MDSDE), MSDE and MDSDE are used to process the sun wheel fault signal, the planet wheel fault signal, the ring gear fault signal, and the health state signal of the planetary gearbox. The results are shown in Figs. 5 and 6, respectively. As can be seen from Fig. 5, MSDE can distinguish sun wheel fault signals from planetary ones. For internal gear ring fault signals and health state signals, the entropy values are mixed together at partial scales and cannot be effectively differentiated. It can be seen from Fig. 6 that MDSDE can effectively distinguish the healthy states of planetary gearbox signals, thus verifying that the improved MDSDE has certain advantages over the traditional method.

However, MDSDE still has certain deficiencies. First, there are partial scale faults that cannot be effectively differentiated; second, MDSDE of the same health state has relatively large fluctuation, so it is difficult to obtain the stationary entropy change curve. The main reason for the above problems may be the possible misjudgment caused by the randomness of the signal. The average calculation of entropy value can effectively avoid the above problems with the relevant knowledge of mathematical statistics. 


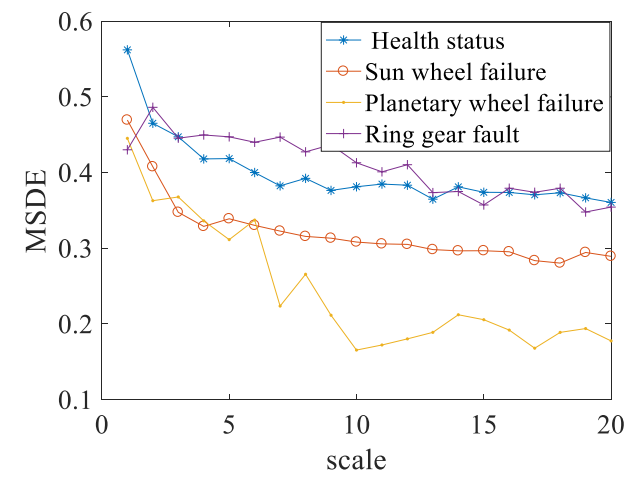

Fig. 5 Multiscale symbolic dynamics entropy

In this paper, the Average Multi-scale Double Symbolic Dynamic Entropy (AMDSDE) is selected, and the main ideas are mainly divided into the following four steps:

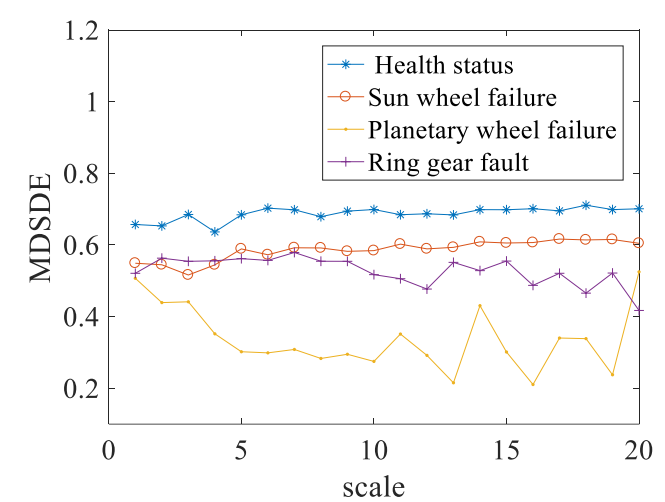

Fig.6 Multiscale double symbolic dynamics entropy

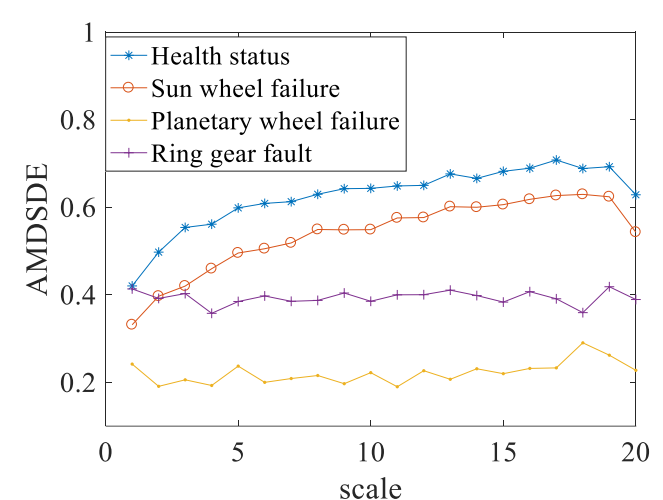

Fig. 7 Average multi-scale double symbolic dynamic entropy

(1) The original time domain signal is segmented by coarse granulation; the coarsegrained segmentation method is the same as 3.3.1.

(2) Use MSDE to calculate all the scale time-domain signals $\left\{y_{j}^{\tau}\right\}$ after coarse-grained segmentation.

$$
\operatorname{MSDE}\left(y_{j}^{\tau}, s, m, \lambda, \tau\right)=\operatorname{SDE}\left(y_{j s}^{\tau}, s, m, \lambda\right)
$$

(3) The average value of each single scale is treated and taken as the entropy value of the scale.

$$
\operatorname{DSDE}(Z, s, m, \lambda, \tau)_{i}=\frac{1}{s} \sum_{i=1}^{s} \operatorname{MSDE}\left(y_{j}^{\tau}, s, m, \lambda, \tau\right)
$$

(4) Calculate the DSDE of each scale, and the AMDSDE is finally obtained.

$$
\operatorname{AMDSDE}(Z, s, m, \lambda, \tau)=\left\{\operatorname{DSDE}(Z, s, m, \lambda, \tau)_{i}, i=1,2, \mathrm{~L}, \tau\right\}
$$

The proposed AMDSDE is used to analyze the simulated signals of the planetary gearbox in four health states, respectively. The entropy values obtained are shown in Fig. 7. The comparison between Fig. 7 with Fig. 6 shows that the method proposed in 
this paper can effectively distinguish the planetary gearbox faults of various health states, and the deficiencies of MDSDE have been well improved.

3.3.3 Parameter Selection of the Multi-scale Double Symbolic Dynamic Entropy

When AMDSDE is used to extract the fault features of vibration signals of planetary gearbox, the main parameters of AMDSDE include the sign number $\mathrm{S}$, embedding dimension $\mathrm{M}$, time delay $\lambda$ and scale factor $\tau$. The multiple regression analysis is used to study the influence of each parameter on AMDSDE. Finally, the symbol number $\mathrm{S}$ $=8$, embedding dimension $M=3$, time delay $\lambda=1$, and scale factor $\tau=20$ are selected.

\section{Experimental Signal Analysis of Planetary Gearbox Sun Wheel Failure}

To verify the effectiveness of the proposed method, experimental signals from different health states of the sun wheels of the planetary gearbox were tested and analyzed on the synthesis test rig for power transmission fault diagnosis (Spectra Quest product in the United States), as shown in Fig. 8. The platform consists of a speed control device, a driving motor, bearing pedestals, a two-stage planetary gearbox, a fixed-shaft gearbox, and a load device. In this paper, four working conditions of the sun wheels of the planetary gearbox, such as health state, broken teeth, wear and crack, are analyzed. The key parameters of the data collected by the test bed are shown in Table 5 .

Table 4 Multiple regression analysis of the influence of parameters on AMDSDE

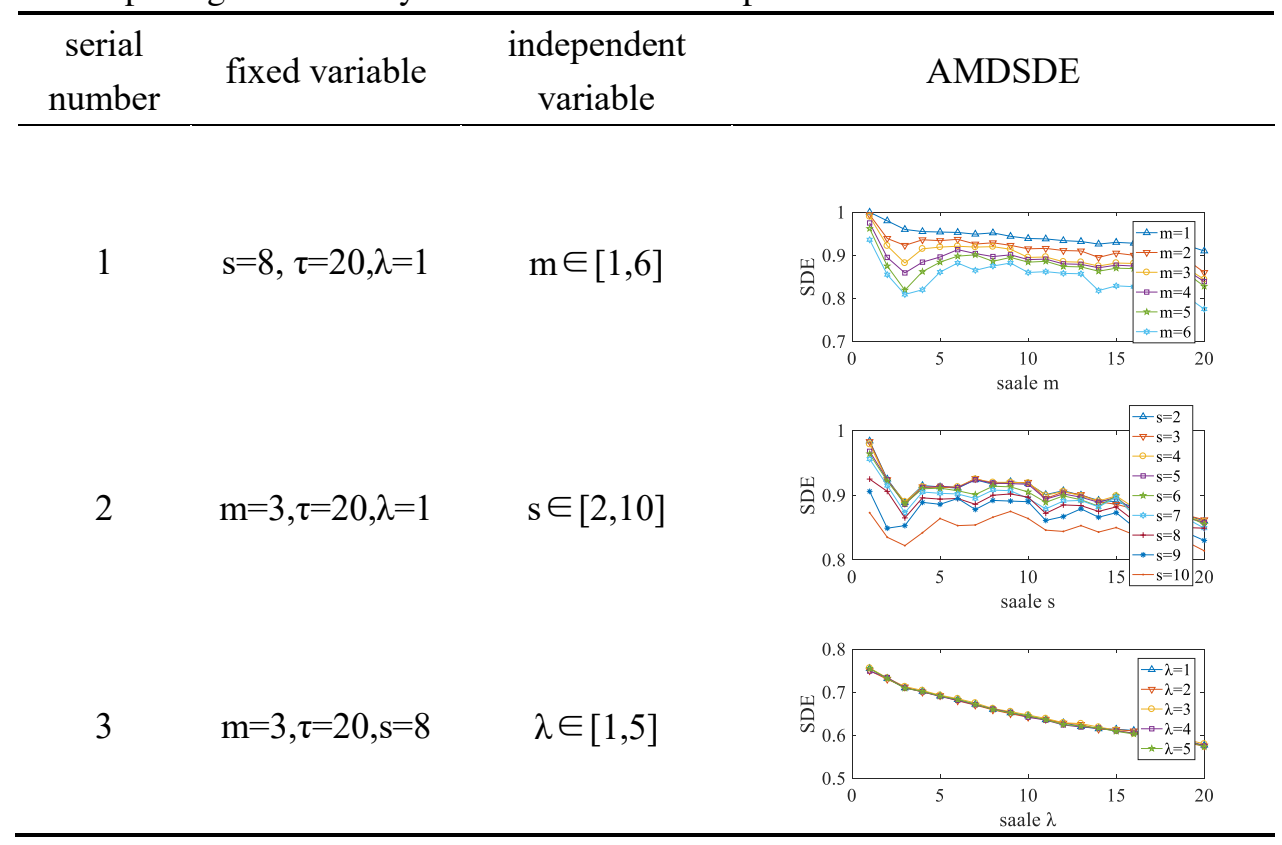

Table 5 Relevant parameters of the experimental signal

\begin{tabular}{cccccc}
\hline type & $\begin{array}{c}\text { sampling } \\
\text { frequency } \\
(\mathrm{Fs})\end{array}$ & $\begin{array}{c}\text { maximum } \\
\text { speed of input } \\
\text { shaft }(\mathrm{ns})\end{array}$ & $\begin{array}{c}\text { maximum } \\
\text { engagement } \\
\text { frequency (fm) }\end{array}$ & $\begin{array}{c}\text { equivalent fault } \\
\text { characteristic frequency } \\
\text { of sun wheel (fs) }\end{array}$ & $\begin{array}{c}\text { sampling } \\
\text { frequency } \\
(\mathrm{t})\end{array}$ \\
\hline data & $5120 \mathrm{~Hz}$ & $1800 \mathrm{r} / \mathrm{min}$ & $600 \mathrm{~Hz}$ & 2.4 order & $10 \mathrm{~s}$ \\
\hline
\end{tabular}

(1) The experimental signal is variable speed signal, and the traditional fault feature 
extracted method will produce frequency ambiguity. In order to solve this problem, the paper first applied the Turn Domain Resampling (TDR) proposed by our group ${ }^{[11]}$ to equivalently transform the signals with variable speed to the four health status signals collected. The original time domain signals and the resampled signals are shown in Fig. 9 and Fig. 10 respectively. In both figures, (a) is the health state signal of the sun wheel, (b) is the broken tooth fault signal of the sun wheel, (c) is the wear fault signal of the sun wheel, and (d) is the crack fault signal of the sun wheel.

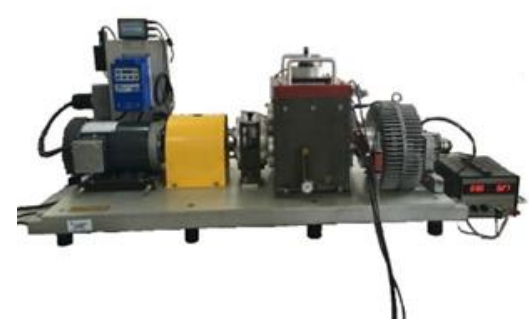

Fig.8 Power transmission fault diagnosis comprehensive test bench

(2) The VMSMD proposed in this paper is used to de-noise the four groups of signals with different fault types after resampling, and then the envelope demodulation of the resampling signal is carried out. The processing results are shown in Fig. 11, in which the four figures are the order envelope spectra of the health state, broken tooth fault, wear fault and crack fault of the sun wheel, respectively.

(a)

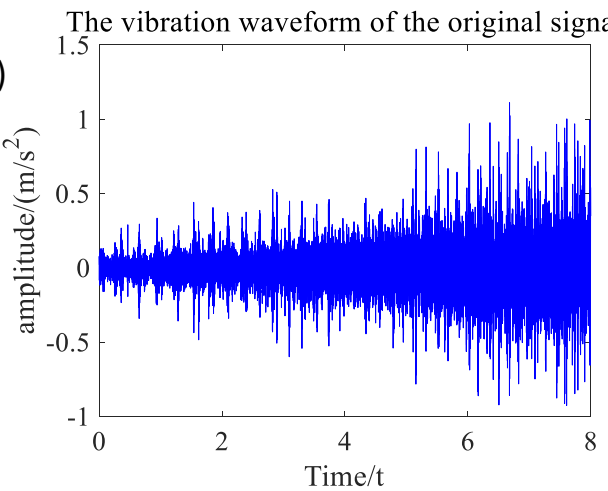

(c)

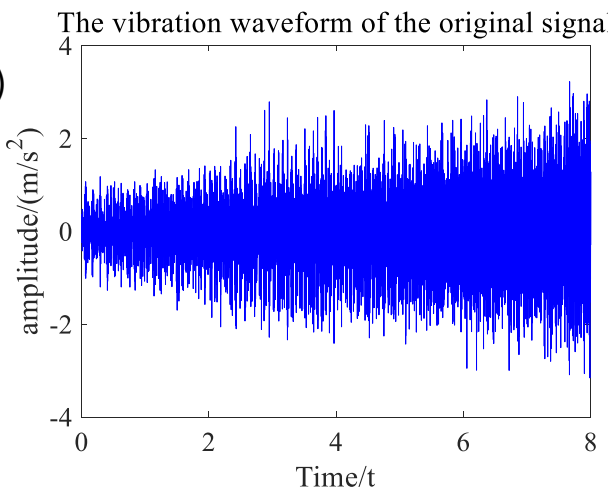

(b)

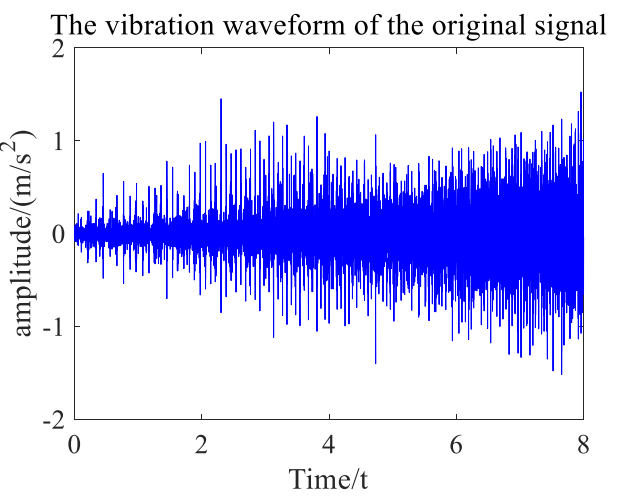

(d)



Fig. 9 Time domain signals of various states of planetary gearbox sun wheel. (a) The sun wheel health state signal (b) The sun wheel broken tooth fault signal(c) The sun wheel wear fault signal

(d) The sun wheel crack fault signal 

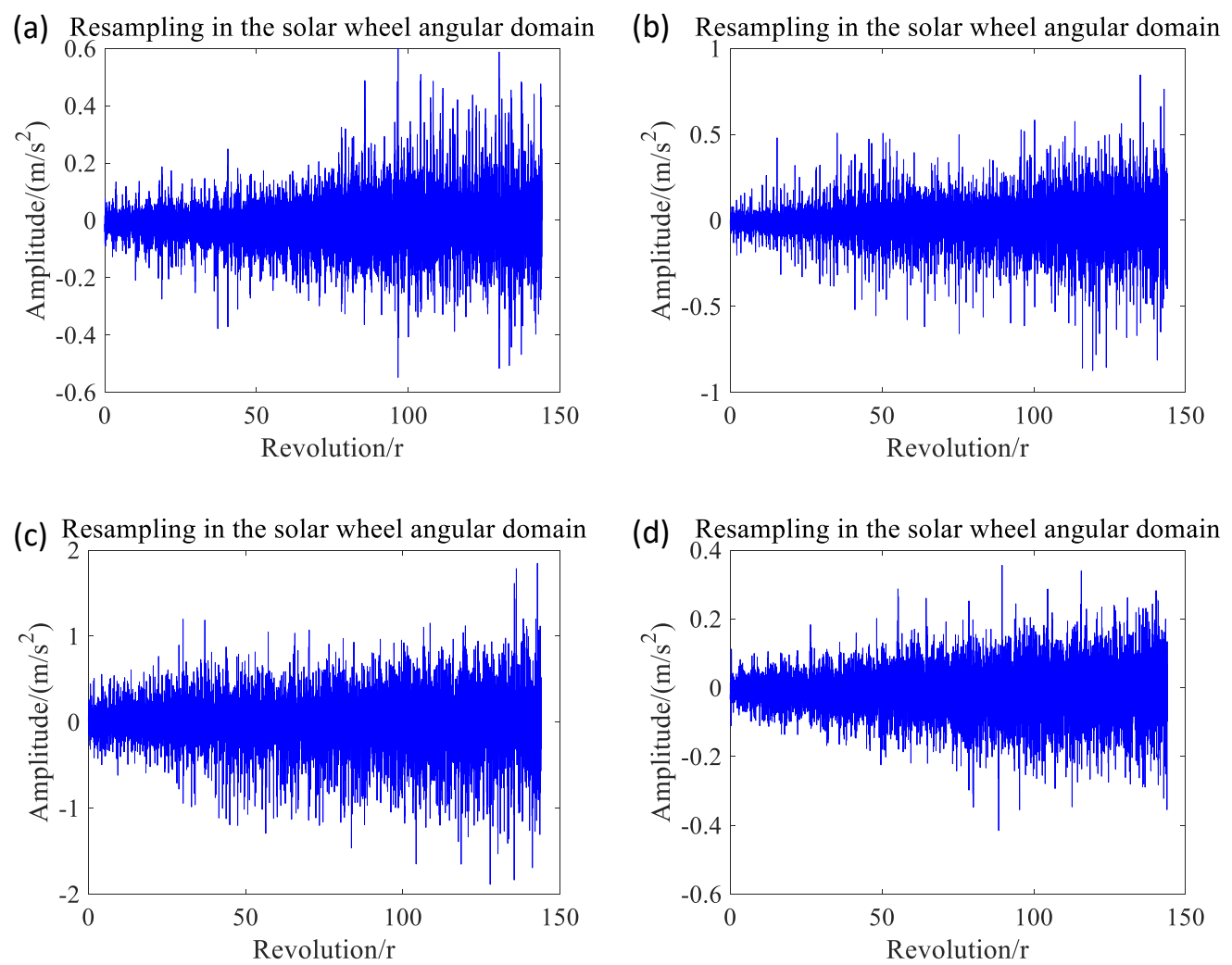

Fig. 10 Resampling signals of the sun wheel in each state of the planetary gearbox. (a) The sun wheel health state signal (b) The sun wheel broken tooth fault signal(c) The sun wheel wear fault signal (d) The sun wheel crack fault signal
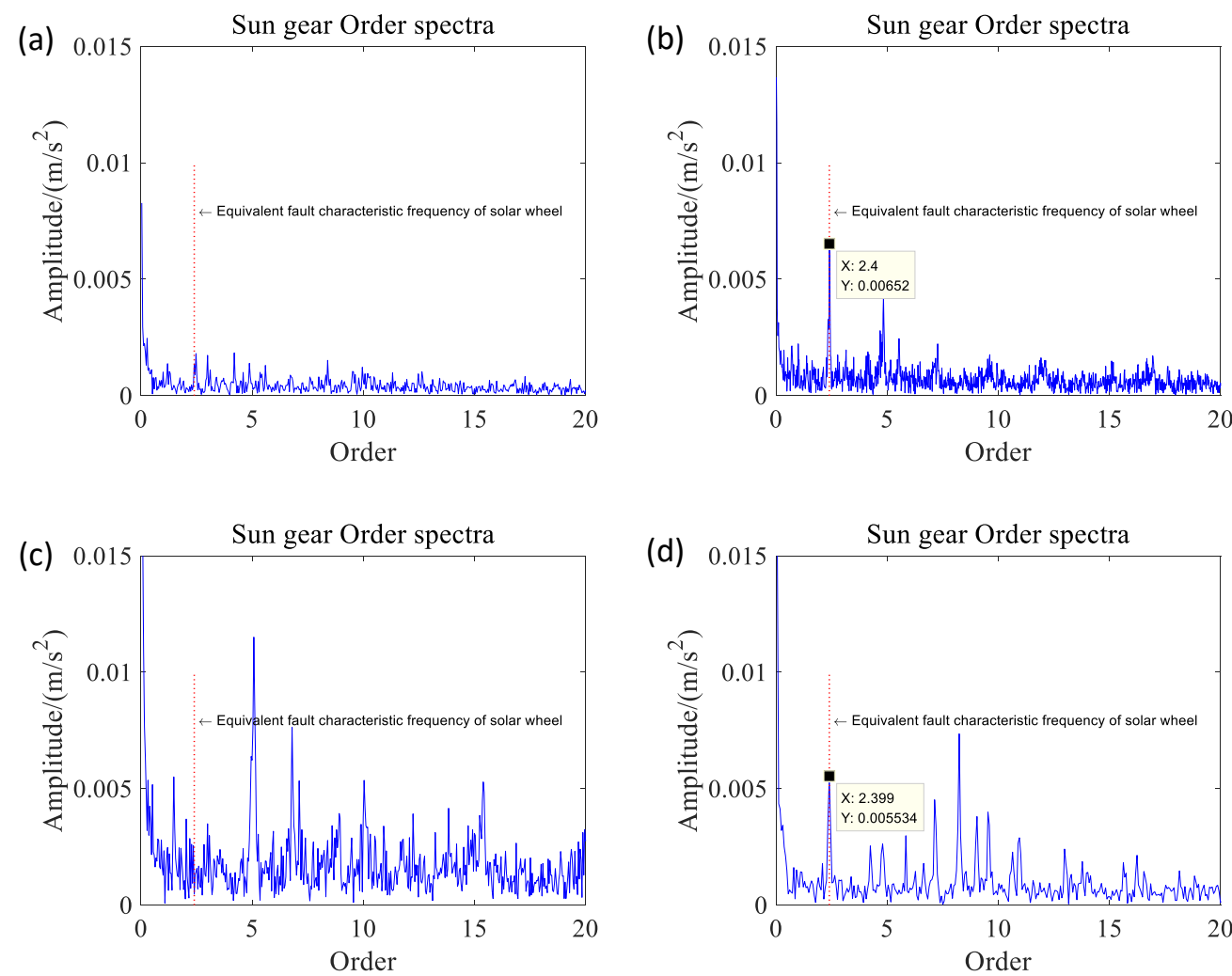

Fig. 11 State order spectra of planetary gearbox sun wheel. (a) The order envelope spectrum of the 
health state (b) The order envelope spectrum of broken tooth fault(c) The order envelope spectrum of wear fault (d) The order envelope spectrum of crack fault

It can be seen from Fig. 11, both the equivalent fault characteristic frequencies of the broken teeth and the cracked states of the sun wheel are 2.4 order after resampling in the angular domain, and the two working conditions cannot be effectively separated by fault characteristic frequencies alone. Since the wear condition is the uniform wear of each gear of the sun wheel, and the fault characteristics are similar to the health characteristics, it is difficult to effectively classify the two conditions only by the fault feature frequency extraction.

(3) In order to distinguish the faults of the four states of the sun wheel effectively, the paper used AMDSDE method to calculate the entropy of the four groups of different fault signals after noise reduction. In order to reflect the advantages of AMDSDE, the traditional MSDE and MDSDE of fault signals in four states are compared respectively. The symbolic number $S=8$, the embedding dimension $M=3$, the time delay $\lambda=1$, and the scale factor $\tau=20$ are chosen when calculating the entropy. The calculated results are shown in Fig. 12-14.

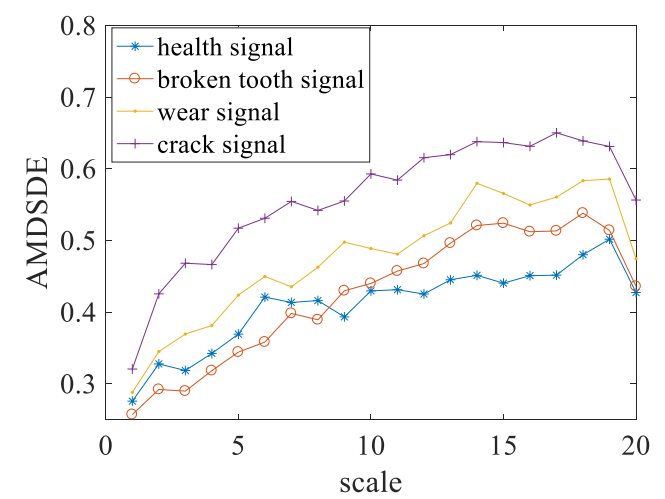

Fig. 12 Average multi-scale double symbol dynamic entropy of sun wheel fault signal



Fig. 13 Multi-scale double symbol dynamic entropy of sun wheel fault signal

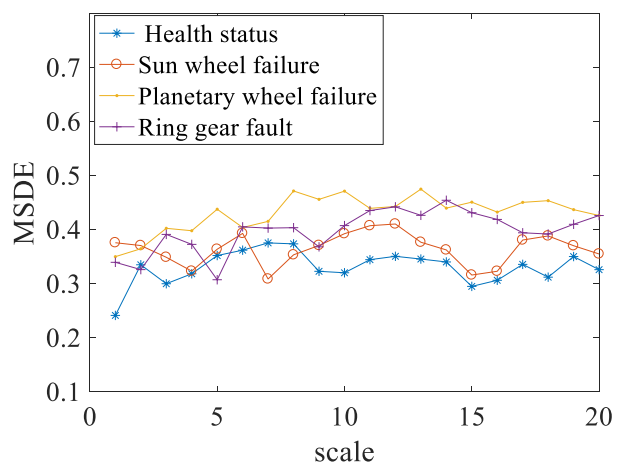

Fig. 14 Multi-scale symbol dynamic entropy of sun wheel fault signal

Fig. 12 shows the AMDSDE entropy values of the planetary gearbox under four working conditions. It can be seen that these values are effectively distinguished. Fig. 13 shows the calculated MDSDE of planetary gearbox under four working conditions, according to which, this algorithm can distinguish the crack state and wear state, but the entropy values of the other two states are mixed together. Fig. 14 is the characteristic 
calculation of each state signal using the traditional MSDE. As can be seen from the figure, in addition to the crack fault, the MSDE values of the other three health states cannot be distinguished. The comparison of these figures shows that the classification effect of MDSDE has been improved compared with MSDE, but local confusion hasn't been addressed yet. Compared with MDSDE and MSDE, AMDSDE has the best performance, which verifies the effectiveness of the proposed method for fault feature classification of planetary gearbox.

\section{Conclusions and Prospects}

\subsection{Conclusions}

Based on the traditional multi-scale morphological filtering the VMSMF is proposed in this paper. The Chebyshev window that is more suitable for the signal of planetary gearbox is selected as the structural element. In addition, an AMDSDE method based on MSDE is proposed to address the difficulty in accurate distinguishing the faults of the sun gear by only depending on frequency. The signals of the four health states of the planetary gearbox sun wheel are used to test the accuracy of the proposed method. These analysis results verified the effectiveness and practicability of the proposed method. The main innovations of this paper are as follows:

(1) The Chebyshev window is selected as the structural element of morphological filtering for the complex structure and severe noise interference of vibration signal of planetary gearbox. VMSMF is proposed to address the problem of low computational efficiency. The method selected more specific structural scales for specific shocks in the signal. The computational efficiency is improved several times, and the more points, the more obvious the advantage.

(2) The DSDE is proposed, which is able to consider the influence of the preposition and post-position symbols of the state mode vector on its state mode probability in the calculation of SDE. DSDE has more advantages over SDE in feature classification when many factors are involved.

(3) Considering that the MDSDE may be misjudged due to the jump of entropy value caused by disturbance in the calculation, this paper proposed AMDSDE. It can effectively avoid the misjudgment caused by local disturbance and has better robustness.

(4) The signals of four different health states of the sun wheel were calculated and analyzed respectively based on VMSMF and AMDSDE proposed in this paper, and good results are obtained.

\subsection{Prospects}

The method proposed in this paper can distinguish different types of health conditions, but it is still unable to identify specific fault types. Therefore, the AMDSDE and deep learning algorithm will be combined to achieve qualitative analysis of signals in the future.

Funding: This research is funded by the National Natural Science Foundation of China (Grant Nos. 52075008).

Conflicts of interest/Competing interests: The content of this paper does not involve any conflict of interest. 
Availability of data and material: The data used in this paper are all owned by the lab of the research group. As the research is still continuing, the data involved in this paper is not publicly available.

Code availability: The algorithm involved in this paper is still being studied by the research group, so it is not publicly disclosed.

Ethics approval: The team affirmed that all the research was original research of the research group without any bad behavior.

Consent to participate: The methods involved in this paper are agreed to be shared. Consent for publication: The content of this thesis is agreed to be published in the journal.

Authors' contributions: L,L. C. and T,T. L. conceived and designed the experiments. J,Y. Z. and T,T.L. performed the experiments. T,T. L. and C.Z. analyzed the data; L,L. C. and J,Y.Z. provided guidance and recommendations for research; T.L. contributed to the contents and writing of the manuscript. All authors have read and approved the final manuscript.

Acknowledgments: The authors would like to acknowledge the support of National Natural Science Foundation of China (grant nos. 52075008) and the Key Laboratory of Advanced Manufacturing Technology. Finally, the authors would like to thank the editors and reviewers for their valuable comments and constructive suggestions.

\section{Reference}

[1] Li Y.B., Feng K., Liang X.H., Zuo M.J. A fault diagnosis method for planetary gearboxes under non-stationary working conditions using improved Vold-Kalman filter and multi-scale sample entropy [J]. Journal of Sound and Vibration. 2019. 439, 271-286.

[2] Zhang M., Jiang Z., Feng K. Research on variational mode decomposition in rolling bearings fault diagnosis of the multistage centrifugal pump [J]. Mech. Syst. Signal Process. 93 (2017) 460-493.

[3] Ren X.P., Li P., Wang C.G., Zhang C. Rolling bearing early fault diagnosis based on improved VMD and envelope derivative operator [J]. Journal of Vibration and Shock.2018. 37(15):6-13.

[4] Serra J P. Image Analysis and Mathematical Morphology [J]. Biometrics, 1982, 39(2):536.

[5] Nikolaou N G, Antoniadis I A. Application of morphological operators as envelope extractors for impulsive-type periodic signals [J]. Mechanical Systems and Signal Processing, 2003, 17(6): 1147-1162.

[6] Wang J., Xu G., Zhang Q., et al. Application of improved morphological filter to the extraction of impulsive attenuation signals [J]. Mechanical Systems and Signal Processing, 2009, 23(1):236-245.

[7] Shen C.Q., Xie W.D., Zhu Z. K. Rolling element bearing fault diagnosis based on EEMD and improved morphological filtering method [J]. Journal of Vibration and Shock, 2013, 32(2):3943.

[8] Jiang W., Zheng Z., Zhu Y., et al. Demodulation for hydraulic pump fault signals based on local mean decomposition and improved adaptive multiscale morphology analysis $[\mathrm{J}]$. Mechanical Systems \& Signal Processing, 2015, s 58-59:179-205.

[9] Li B., Zhang P.L., Wang Z.J., et al. Gear fault detection using multi-scale morphological filters [J]. Measurement, 2011, 44(10):2078-2089.

[10] Chen Q., Chen Z., Sun W., et al. A new structuring element for multi-scale morphology analysis 
and its application in rolling element bearing fault diagnosis [J]. Journal of Vibration and Control, 2015, 21(4):765-789.

[11] Liu T.T., Cui L.L., Zhang C. Study on fault diagnosis method of planetary gearbox based on turn domain resampling and variable multi-scale morphological filtering [J]. Symmetry. 2021. 52(13), 1-17

[12] Claude E. Shannon. A mathematical theory of communication [J]. The Bell System Technical Journal. 1948.5(1):1-53

[13] Kolmogorov A N. A new metric invariant of transient dynamical systems and auto-morphisms in Lebesgue spaces [J]. Dokl Akad Nauk SSSR, 1958, 951(5): 861-864.

[14] Pincus S M. Approximate entropy as a measure of system complexity [J]. Proceedings of the National Academy of Sciences, 1991, 88(6): 2297 -2301.

[15] Richman J S, Moorman J.R. Physiological time-series analysis using approximate entropy and sample entropy [J]. Ajp Heart \& Circulatory Physiology, 2000, 278(6): H2039-H2049.

[16] Bandt C., Pompe B. Permutation entropy: a natural complexity measure for time series [J]. Physical Review Letters, 2002, 88(17): 174102.

[17] Daw C.S., Finney C E, Tracy E R. A review of symbolic analysis of experimental data [J]. Review of Scientific Instruments, 2003, 74(2): 915-930.

[18] Hao, B.L. Symbolic dynamics and characterization of complexity [J]. Physica D: Nonlinear Phenomena, 1991, 51(1-3): 161-176.

[19] Zheng W.M., Hao B.L. Applied Symbolic Dynamics [J]. Progress in Physics, 1996, 10(3): 316373.

[20] KURTHS J, VOSS A, SAPATIN P, et al. Quantitative analysis of heart rate variability [J]. Chao, 1995(5):88-94.

[21] Zhang H., Zeng W.T., Yan W. Fault diagnosis of hydraulic pump based on symbolic dynamics entropy and SVM [J]. Journal of Vibration, Measurement \& Diagnosis, 2017, 32(4):288293.

[22] Costa M, Goldberger A L, Peng C K. Multiscale entropy to distinguish physiologic and synthetic RR time series [J]. Computers in Cardiology, 2002, 29(29): 137-140.

[23] Ding C., Feng F.Z., Zhang B.Z., Wu S.J. MMSDE and its application in feature extraction of a planetary gearbox [J]. Journal of Vibration and Shock. 2020, 39(13):97-102.

[24] Li Y.B., Yang Y.T., Li G.Y., et al. A fault diagnosis scheme for planetary gearboxes using modified multi-scale symbolic dynamic entropy and mRMR feature selection [J]. Mechanical Systems and Signal Processing, 2017, 91:295-312.

[25] Zhao H.M.; Liu H.D., Xu, J.J. Performance Prediction Using High-Order Differential Mathematical Morphology Gradient Spectrum Entropy and Extreme Learning Machine. IEEE TRANSACTIONS ON INSTRUMENTATION AND MEASUREMENT.2020. 69(7), 41654172

[26] Guo J.C.; Zhen D.; Li, H.Y. Fault detection for planetary gearbox based on an enhanced average filter and modulation signal bispectrum analysis. ISA TRANSACTIONS. 2020. 101, 408-420.

[27] N. G. Nikolaou, I. A. Antoniadis. Application of morphological operators as envelope extractors for impulsive-type periodic signals [J]. Mechanical Systems and Signal Processing. 2003. 17(6), 1147-1162.

[28] Maragos, P. Representations for Morphological Image Operators and Analogies with Linear Operators [J]. Advances in imaging and electron physics. 2013. 177, 45-187. 
[29] Costa M., Goldberger A. L., Peng C. K. Multiscale entropy analysis of complex physiologic time series [J]. Physical Review Letters, 2002, 92(8): 705 -708. 